\title{
How should a PI justify the use of vertebrate species when asked about invertebrate alternatives?
}

\author{
Jerald Silverman, DVM
}

As part of his most recent IACUC submission, Dr. John Tuckerton requested the use of $40 \mathrm{C} 57 \mathrm{Bl} / 6$ mice to determine the most effective in vivo dose of P4484-17, a newly synthesized molecule that, in vitro, exhibited strong inhibition of prostaglandin $E_{2}$ $\left(\mathrm{PGE}_{2}\right) \cdot \mathrm{PGE}_{2}$ is an activator of the Wnt signaling pathway, a protein-based pathway that can impact embryonic development. The experimental design was to test three dose levels of P4484-17, with ten mice per dose level and one group of ten untreated control mice. Based on the dose that led to the greatest inhibition of $\mathrm{PGE}_{2}$ in the mice, Tuckerton would initiate a much larger study of the Wnt pathway and embryonic development. The larger study involved nearly 1000 mice and examined multiple aspects of mouse embryonic development, with a focus on how the longitudinal (anteroposterior) axis of the body developed.

One of the reviewers of Tuckerton's protocol submission was Dr. Andrea McCarthy, a geneticist who was aware that there was a Wnt signaling pathway in fruit flies. One of her written questions to Tuckerton was:
Can this study be performed in fruit flies? Tuckerton responded that his goal was to be able to translate his work on mouse development to human development and therefore, in his opinion, using an invertebrate model was not appropriate.

Do you think that Tuckerton's answer to McCarthy's question was sufficient to justify his use of mice? Whether you agree or disagree with Tuckerton's response, what additional questions would you ask of Tuckerton, based on the limited information presented in this scenario?

\section{RESPONSE}

\section{It's all in the questions}

\section{Marcy Brown ${ }^{1}$ \& Deb Frolicher ${ }^{2}$}

US Government Principle III (ref. 1), the Guide for the Care and Use of Laboratory Animals (Guide) ${ }^{2}$, and the Animal Welfare Act regulations (AWARs) ${ }^{3}$ all require justification or rationale for the appropriateness of the species proposed for an animal use activity. It is the IACUC's responsibility during review of protocols and significant changes to protocols, to consider the adequacy of this justification.

The Guide also directs the Principal Investigator (PI) to consider, and the IACUC to review, "the appropriateness of the use of other species, isolated organ preparation, cell or tissue cultures, or computer simulation." Therefore, McCarthy was fulfilling her responsibility as an IACUC reviewer to pose a question regarding the use of another species, including an invertebrate model.

While the IACUC needs to be assured that the species justification is sufficient, they must also be prudent in their examination of the scientific merit of the proposed work. As stated in the Guide, "while the responsibility for scientific merit review normally lies outside the IACUC, the committee members should evaluate scientific elements of the protocol as they relate to the welfare and use of animals." We suggest that species justification, as presented in this scenario, can and does relate directly to the prevention of unnecessary animal use. Without knowing how or whether this work is funded, it is not possible to know if peer review of the scientific merit was conducted. In lieu of a formal peer review, the IACUC should be reminded that they may invite outside consultants to provide input during review, including the appropriateness of the species ${ }^{4}$.

Was Tuckerton's response a sufficient justification for his use of mice? Given the plethora of literature describing the significant contributions made by fruit flies (Drosophila melanogaster) to the understanding of human diseases, including developmental and neurological disorders ${ }^{5}$, Tuckerton's response that results using an invertebrate would not translate to human development was not sufficient justification.
However, given the simplistic nature of McCarthy's question, "Can this study be performed in fruit flies?" Tuckerton likely considered his response to be appropriate.

When requesting additional information from investigators, IACUCs should strive to ask detailed and specific questions. A better question may have been "Much of what is known about the Wnt signaling pathways comes from genetic studies in Drosophila ${ }^{6}$. Describe why studies assessing PGE2 activation in the Wnt signaling pathway cannot be conducted in fruit flies prior to work in mice."

Given the limited information in this scenario, perhaps the institution's protocol form did not request species justification in a way that elicited a complete response. Many forms simply state "Justify the use of the species requested." Asking a more detailed question such as "Provide scientific justification for the species requested, addressing why animals from a 'lower' species, non-animal alternatives, or computer simulation cannot be used to achieve the desired results", may avoid the need for additional information.

In conclusion, an evaluation of the species justification must be incorporated 